\title{
Turbulence Control in Wall-Bounded Flows by Spanwise Oscillations
}

\author{
R. AKHAVAN, W. J. JUNG and N. MANGIAVACCHI \\ Department of Mechanical Engineering \\ The University of Michigan, Ann Arbor, MI 48109-2125, USA
}

\begin{abstract}
The feasibility of control of wall turbulence by high frequency spanwise oscillations is investigated by direct numerical simulations of a planar turbulent channel flow subjected either to an oscillatory spanwise crossflow or to the spanwise oscillatory motion of one of the channel walls. Periods of oscillation, $T_{o s c .}^{+}=T_{o s c .} u_{\tau}^{2} / \nu$, ranging from 25 to 500 were studied. For $25<T_{o s c .}^{+}<$ 200 production of turbulence is suppressed. The most effective suppression of turbulence occurs at $T_{o s c}^{+}=100$, for which the overall turbulence production is reduced by $62 \%$ compared to the unperturbed channel and sustained turbulent drag reductions of $40 \%$ are obtained. The suppression of turbulence is due to a continual shift of the near wall streamwise vortices relative to the wall layer streaks, which in turn leads to a widening, merging and weakening of the wall layer streaks and an overall reduction in the turbulence production. The turbulence suppression mechanism observed in these studies opens up new possibilities for effective control of turbulence in wall-bounded flows.
\end{abstract}

Key words: turbulence control - drag reduction - boundary layer flows

\section{Introduction}

Research on wall flow turbulence over the past twenty years has revealed that the production of turbulence in wall-bounded flows is accomplished by organized structures in the near wall region in a sequence of repeatable and quasi-periodic events. The emergence of such a structural picture has opened up the possibility of control of turbulence in wall-bounded flows, potentially leading to significant reductions in the turbulent skin-friction drag.

In this study, we explore the feasibility of control of wall turbulence by superimposed spanwise oscillations. The studies were motivated by results from a number of recent experimental and numerical investigations $[2,3,6]$ which have shown that when a two-dimensional boundary layer is suddenly subjected to a transverse pressure gradient or a crossflow, the flow experiences temporary reductions in its turbulence level. The objective of the present study is to determine whether it is possible to achieve a sustained suppression of turbulence in wall flows by keeping the turbulence from ever reaching an equilibrium state, using spanwise oscillations. The studies are based on results from direct numerical simulations. A brief account of some of the results reported here has been previously given in [4]. 


\section{Numerical Methods}

Direct numerical simulations were performed of a fully developed planar turbulent channel which was subjected either to an oscillatory spanwise crossflow (equivalently a spanwise pressure gradient) or to the spanwise oscillatory motion of one of the channel walls. Computations were done using standard Fourier/Chebyshev pseudospectral methods [1], implemented in parallel on a 32-node iPSC/860 Intel hypercube [5]. A fully-developed turbulent flow at a mean Reynolds number of 3000 based on half channel width and bulk velocity $\left(R e_{\tau}=200\right)$ was established in the channel by perturbing an initially laminar state with a combination of twoand three-dimensional least stable eigenmodes of the Orr-Sommerfeld equation, and carrying out the simulations until the flow had reached a stationary turbulent state with one- and two-point statistics in good agreement with known results. The computational domain was $1.6 \pi h$ (1010 wall units) long in each of the streamwise $(\mathrm{x})$ and spanwise (z) directions and $2 h$ wide in the normal $(\mathrm{y})$ direction, and calculations were done with $64 \times 129 \times 128$ de-aliased modes in the $\mathrm{x}, \mathrm{y}$ and $\mathrm{z}$ directions, respectively, to resolve all the essential scales of the turbulent motion.

The influence of an additional spanwise strain on the dynamics of the turbulent state described above was then studied. The oscillations were generated either by a spanwise crossflow with specified flow rate per unit width equal to $Q_{z}=(0.4$ or 0.8 or 1.2) $Q_{x} \sin \omega t$, or by the motion of one of the channel walls according to $W_{\text {wall }}=0.8\left(Q_{x} / 2 h\right) \sin \omega t$. The flow rate per unit width in the streamwise direction $Q_{x}$ was kept fixed at the unperturbed turbulent channel value in all the runs. Calculations were made for non-dimensional periods of oscillation $T_{\text {osc. }}^{+}=$ $T_{\text {osc. }} u_{\tau}^{2} / \nu$ ranging from 25 to 500 , where $u_{\tau}$ is the wall friction velocity in the unperturbed channel.

\section{Results}

The time evolution of the streamwise component of the wall shear stress subsequent to the start of oscillations is shown in Figure 1. With the exception of the case $100 \mathrm{~W}$, in all the runs shown in Figure 1 the oscillations were generated by a crossflow with flow rate per unit width equal to $0.8 Q_{x} \sin \omega t$. The case $100 \mathrm{~W}$ demonstrates the effect of oscillation of one of the channel walls with velocity $W_{\text {wall }}=0.8\left(Q_{x} / 2 h\right) \sin \omega t$ and period $T_{o s c .}^{+}=100$.

As seen in Figure 1, oscillations with $25 \leq T_{o s c}^{+} \leq 200$ result in reductions of 10 to $40 \%$ in the streamwise wall shear stress. The size of the reduction is strongly dependent upon the oscillation frequency. The largest drop is obtained at $T_{o s c .}^{+}=100$, for which the streamwise wall shear stress is reduced by $40 \%$ compared to the unperturbed turbulent channel. These reductions are not transient phenomena, but are sustained in the long term after the flow has reached a statistically periodic steady state. The reductions are independent of whether the oscillations are produced by a crossflow or by the motion of one the channel walls, 


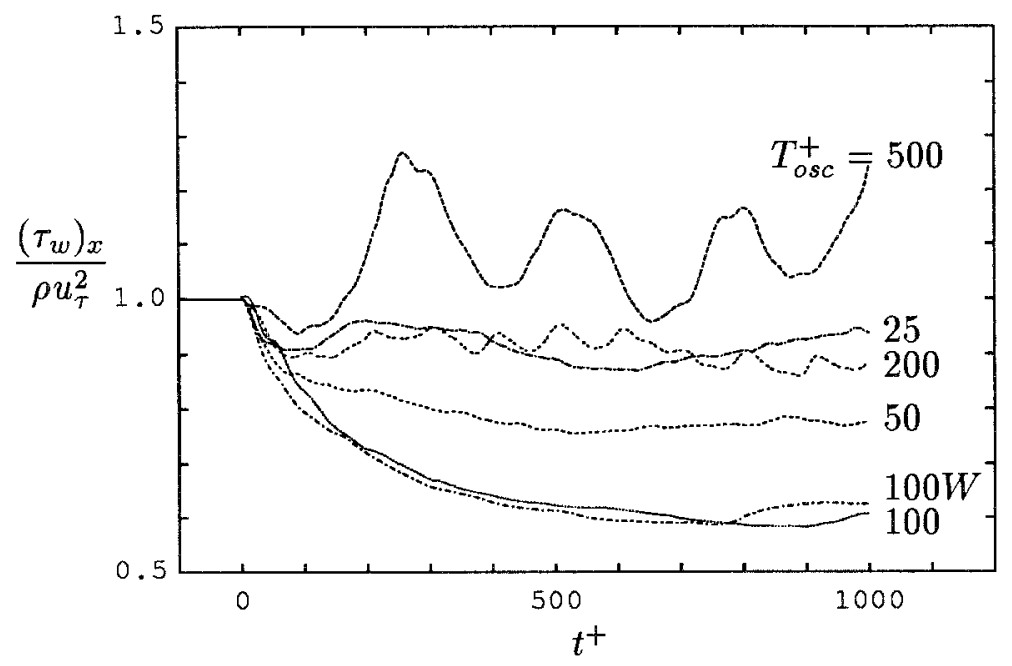

Fig. 1. Time evolution of the streamwise wall shear stress subsequent to the start of oscillations at various frequencies.

as can be seen from a comparison of the two curves 100 and 100W in Figure 1. For oscillations produced by the motion of a channel wall, however, the reduction in turbulence activity is restricted only to the channel half which is adjacent to the oscillating wall. The flow in the other channel half remains fully turbulent.

A summary of the one-point statistics of the flow in the presence of crossflow oscillations at $T_{o s c}^{+}=100$ and $Q_{z}=0.8 Q_{x} \sin \omega t$ is shown in Figure 2. The shown statistics are from the 9 th period of oscillations, when the flow has reached a statistically periodic steady state. The statistics for $t^{+}=0$, corresponding to the unperturbed channel, and the experimental data of Wei and Willmarth [7] for turbulent flow in a two-dimensional channel are also shown for reference. The oscillations result in a $65 \%$ reduction in the overall turbulence production and comparable reductions in the Reynolds shear stress and the turbulence intensities.

Analysis of the dynamics of the turbulence structures in the presence of the oscillations (Figure 3 ) reveals that the suppression of turbulence observed in these studies is due to a continuous shift of the near wall streamwise vortices relative to the wall layer streaks under the influence of the imposed oscillations. The shift leads to a breakdown of one of the critical links in the feedback mechanism which normally sustains the turbulence in wall flows. The resultant flow is one in which the turbulence structures are much wider and weaker, as shown in figure 3.

These results demonstrate that significant reductions in wall flow turbulence can be achieved by keeping the turbulence from ever reaching an equilibrium state. In the present study, this nonequilibrium state was maintained by spanwise oscillations. This is not the most economical way of suppressing the turbulence if one is concerned about the overall energy savings, since the power savings 
$(a)$

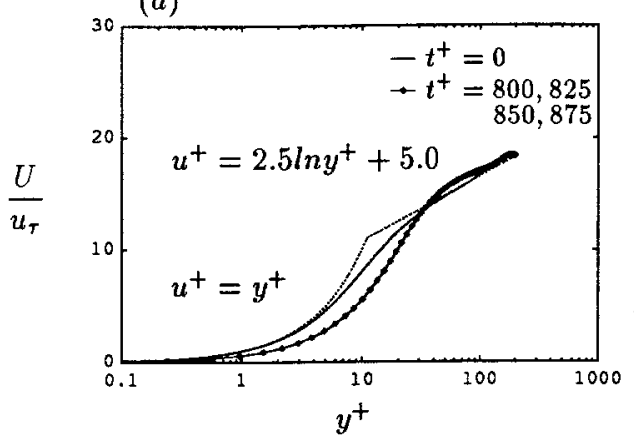

(b)

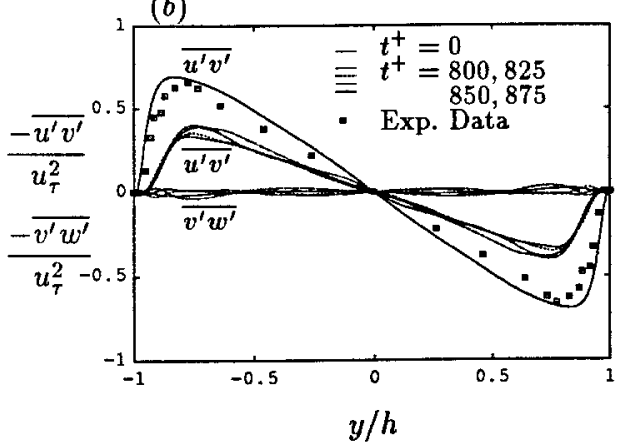

(c)

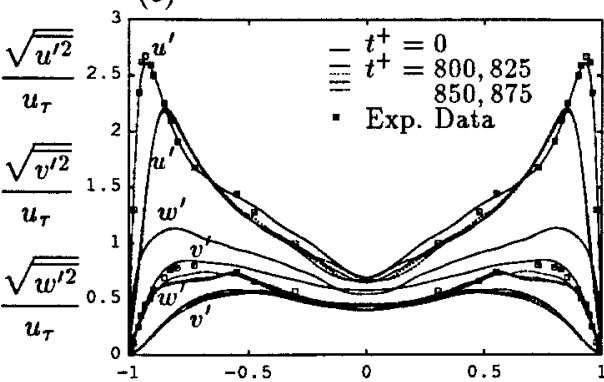

$y / h$

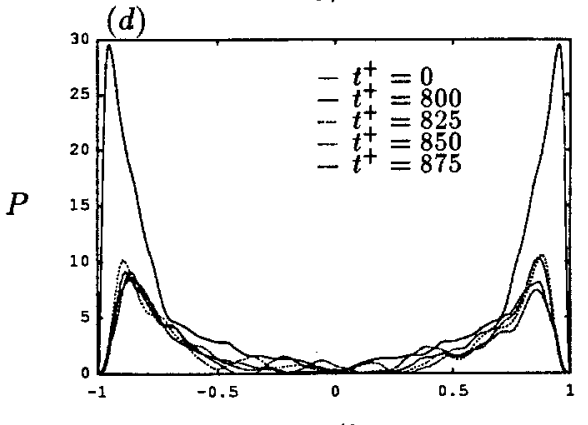

$y / h$

Fig. 2. Profiles of (a) mean streamwise velocity, (b) Reynolds shear stress, (c) turbulence intensities and (d) turbulence production in the presence of crossflow oscillations at $T_{\text {osc. }}^{+}=100$ compared to the unperturbed channel (solid lines).

obtained as a result of the suppression of turbulence is offset by the power required to oscillate the plate. However, it is our belief that the concepts underlying the suppression mechanism observed in these studies can be implemented in novel active and even passive turbulence control schemes in which little or no penalty is paid in way of the power requirements of the control schemes. Efforts along these directions are currently under way.

\section{Acknowledgements}

This work was supported in part by AFOSR grant \#91-0402. The computations were performed on the San Diego Supercomputer Center iPSC/860 Intel hypercube.

\section{References}

1. Akhavan, R., Kamm, R.D. and Shapiro, A.H., An investigation of transition to turbulence in bounded oscillatory Stokes flows- Part 2. Numerical simulations. J. Fluid Mech. 225 (1991) 423-444. 


\section{Unperturbed Channel}

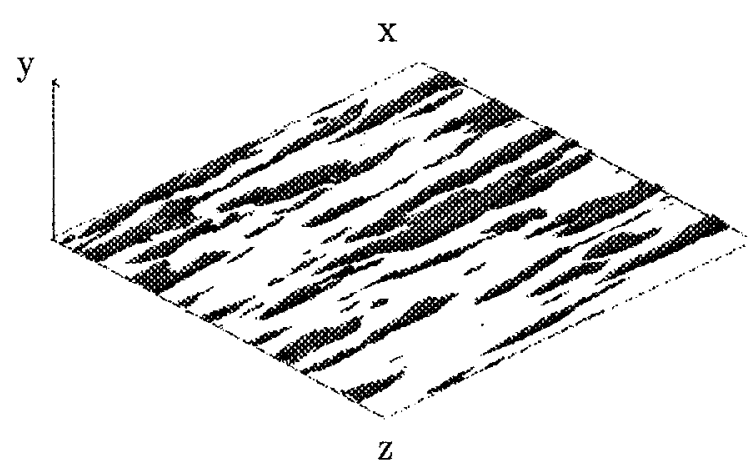

Crossflow Osc. $T^{+}=100\left(t^{+}=825\right)$

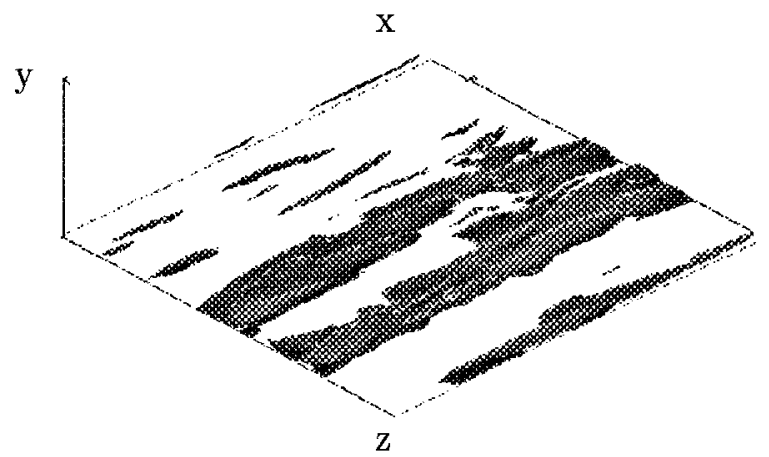

Fig. 3. The high speed streaks in the unperturbed channel compared to the structures in the presence of crossflow oscillations at $T_{\text {osc. }}^{+}=100$. The streaks are captured by contour surfaces of constant spanwise vorticity. The vorticity isosurface represented in each case is $15 \%$ higher than its corresponding average spanwise wall vorticity.

2. Bradshaw, P. and Pontikos, N., Measurements in a turbulent boundary layer on an infinite swept wing. J. Fluid Mech. 159 (1985) 105-130.

3. Driver, D. and Hebbar, S., Experimental study of a three-dimensional, shear-driven, turbulent boundary layer. AIAA J. 25 (1987) 35-42.

4. Jung, W.J., Mangiavacchi, N. and Akhavan, R., Suppression of turbulence in wall-bounded flows by high frequency spanwise oscillations. Phys. Fluids A 2 (1992) 1605-1607.

5. Mangiavacchi, N. and Akhavan, R., Parallel pseudospectral algorithms for direct numerical simulations of turbulence. (submitted)

6. Moin, P., Shih, T., Driver, D. and Mansour, N. Direct numerical simulation of a three-dimensional turbulent boundary layer. Phys. Fluids A 2 (1990) 1846-1853.

7. Wei, T. and Willmarth, W.W., Reynolds number effects on the structure of a turbulent channel flow. J. Fluid Mech. 204 (1991) 57-96. 